www.nature.com/clinicalpractice/uro

GLOSSARY

CYSTOSCOPY

Endoscopy of the urinary

bladder via the urethra with SUI who were in the secretory-phase of their menstrual cycle. This result might be due to a simultaneous increase in TIMP-2 expression, suggesting that elastin metabolism in this group could be altered mostly by elastases rather than MMPs. Elastolytic activity increased in fibroblasts from women with SUI with increasing relaxin concentrations, which could be due to either decreased inhibition by $\alpha-1$ antitrypsin or increased elastase activity.

The authors note that this study was not powered to detect true differences between groups and that these in vitro results may not apply in vivo.

Original article Chen B et al. (2005) Elastin metabolism in pelvic tissues: Is it modulated by reproductive hormones? Am JObstetetrics and Gynecology 192: 1605-1613

\section{Precancerous prostate lesions and serum levels of insulin-like growth factor 1}

A recent study by Nam and colleagues evaluated the possibility of an association between high serum insulin-like growth factor-I (IGF-I) levels and precancerous lesions of the prostate. High serum IGF-I levels have previously been associated with an increased risk of development of prostate cancer.

The authors investigated 308 patients, all of whom had been referred either because of raised serum prostate-specific antigen levels, or an abnormal rectal examination. Following biopsy, 103 patients (the cases) were found to have high-grade prostatic intraepithelial neoplasia (HGPIN) but no adenocarcinoma. The remaining 205 patients (the controls) had no evidence of either HGPIN or adenocarcinoma. In addition to other plasma proteins, the authors measured serum IGF-I levels in the participants before carrying out data analysis comparing the case and control groups.

It emerged that mean serum levels of IGF-I in the case group were significantly higher than those of the control group $(P=0.01)$. Following adjustments, the odds ratio for diagnosis of HGPIN amongst patients in the highest relative to the lowest quartile of serum levels of IGF-I was found to be $1.94(P=0.04)$.

The authors conclude that despite limitations of the study such as the level of statistical significance and small sample size, a possible association emerges between high serum IGF-I levels and the presence of HGPIN. This may help shed light on the basis of the relationship between IGF-I and the risk of prostate cancer.

Original article Nam RK et al. (2005) Serum insulinlike growth factor-I levels and prostatic intraepithelial neoplasia: a clue to the relationship between IGF-I physiology and prostate cancer risk. Cancer Epidemiol Biomarkers Prev 14: 1270-1273

\section{Initial evaluation of women with irritative voiding symptoms}

In a recent study reported in the American Journal of Obstetrics and Gynecology, Sokol and colleagues set out to test the hypothesis that, because of the rarity of abnormal findings, the routine use of urinary cytology and CYSTOSCOPY is of limited value in the initial evaluation of women with irritative voiding symptoms. These tests are currently used to detect urinary tract cancers, but the authors suggested that they may not be necessary in the initial evaluation because of the low prevalence of these cancers in an unscreened population.

The team looked at 1,783 cytology results obtained between January 2000 and July 2003 from women complaining of urinary urgency, painful urination, urinary frequency or nocturia. Only $0.4 \%(n=7)$ of these results were classified as suspicious or malignant. A subset of 564 patients was then selected for cystoscopic analysis. Of these, only one patient was found to have transitional-cell carcinoma. This patient had received a normal cytology result.

The researchers found no association between traditional risk factors for bladder cancer and atypical cytology results, and suggested that atypical cytology may indicate inflammation rather than malignancy.

The authors concluded that urinary cytology and cystoscopy are low-yield tests and are therefore unsuitable for routine use in the initial evaluation of women with irritative voiding symptoms.

Original article Sokol ER et al. (2005) Results of urine cytology testing and cystoscopy in women with irritative voiding symptoms. Am J Obstet Gynecol 192: 1560-1565 